\title{
KINEMATIC STUDY OF FOLDING BRIDGE
}

Lucretia Popa $^{1}$, Iulian Stefan Moise ${ }^{2}$, Valentin Vladut ${ }^{1}$, Luciana Dudici $^{3}$, Vasilica Stefan ${ }^{1}$

${ }^{1}$ National Institute of Research-Development for Machines and Installations Designed to Agriculture and Food Industry, Romania; ${ }^{2}$ Etudes et Productions Schlumberger, France;

${ }^{3}$ University Politehnica of Bucharest, Romania

lucretia_popa@yahoo.com,stef_moise@yahoo.fr,valentin_vladut@yahoo.com, luciana_ionita@yahoo.com, valle_vali@yahoo.com

\begin{abstract}
Over the water courses or irrigation canals different bridges may be built. These constructions can be fixed or movable (folding). Fixed bridges have the great disadvantage of hindering the movement of boats, which are higher than the bridge level, on the watercourses. In order to eliminate this disadvantage, the folding bridges can be created, which allow the opening of the navigation paths when necessary. The bridge is made up of eight segments, one of which is fixed to the base, and the other seven segments fold, so that an octagon is made.
\end{abstract}

Keywords: folding bridge, kinematic analysis.

\section{Introduction}

Folding bridges are of great interest, allowing the navigation of boats along river courses, over which these bridges are located, without restrictions regarding the boat gauge. At present, several folding bridge constructions are known, some even mobile, being located on a means of transport and transported to various areas along the course of the river, depending on the transport needs from one side to the other. Among the most famous and representing real jewels we can enumerate: Rolling Bridge, (England) [1], Slauerhoffbrug, (Netherlands) [2], Scale Lane Footbridge [3], Horn Bridge (Germany) [4], Rhyl Foryd Harbour Bridge-UK [5] etc. The folding mobile bridges can also be transported and located in areas, where agricultural crops are located, to allow agricultural machinery being crossed over the rivers, if needed for agricultural activities in the respective areas. That is why in this article we will deal with the kinematic study of these folding bridges.

In this paper, the kinematic analysis of a mechanism that underlies the construction of such a folding bridge is made.

Hydraulic cylinders are used to move the bridge elements. These hydraulic cylinders are contained in structural modules, called motor dyads. In addition to the motor dyads, the mechanism structure also contains the so-called dyads of aspect 1 (RRR dyad). Computational procedures have been developed in the Matlab syntax for these modules, in order to simplify the kinematic study of the mechanism, meaning to determine the kinematic elements positions and angular and linear velocities, and accelerations. In the structural and kinematic analysis of the folding bridge, methods for solving mechanisms and also numerical methods have been used [6-16]. In order to write the equations necessary for the mechanism analysis, the independent contours method was used $[6 ; 9 ; 10-16]$. To solve the systems of positions' non-linear equations, Newton-Raphson and gradient methods were used, while to solve the systems of linear equations of velocities and accelerations the Gaussian elimination method was used $[7 ; 8 ; 10]$.

\section{Materials and methods}

\section{Structural analysis of the mechanism}

Figure 1 shows the kinematic scheme of a folding bridge consisting of eight segments, one of which is fixed and seven other segments are mobile. Figure 2.a shows the multipolar scheme of the mechanism in Figure 1, while Figure 2.b highlights the multipolar groups the mechanism is made of.

From the multipolar scheme (Figure 2.a) and from the structural relation (Figure 2.b) it can be noticed that the bar mechanism in Figure 1 consists of seven motor dyads: $\operatorname{RRTaR}(1,2,3)$, RRTaR(6,7,8), $\quad \operatorname{RRTaR}(11,12,13), \quad \operatorname{RRTaR}(16,17,18), \quad \operatorname{RRTaR}(21,22,23), \quad \operatorname{RRTaR}(26,27,28)$, $\operatorname{RRTaR}(31,32,33)$, as well as of dyads of aspect 1: $\operatorname{RRR}(4,5), \operatorname{RRR}(9,10), \operatorname{RRR}(14,15), \operatorname{RRR}(19,20)$, $\operatorname{RRR}(24,25), \operatorname{RRR}(29,30), \operatorname{RRR}(34,35)$. 
The kinematic scheme of the folding bridge in folded position is shown in Figure 3. There we can see that, in folded position, the folding bridge forms an octagon.

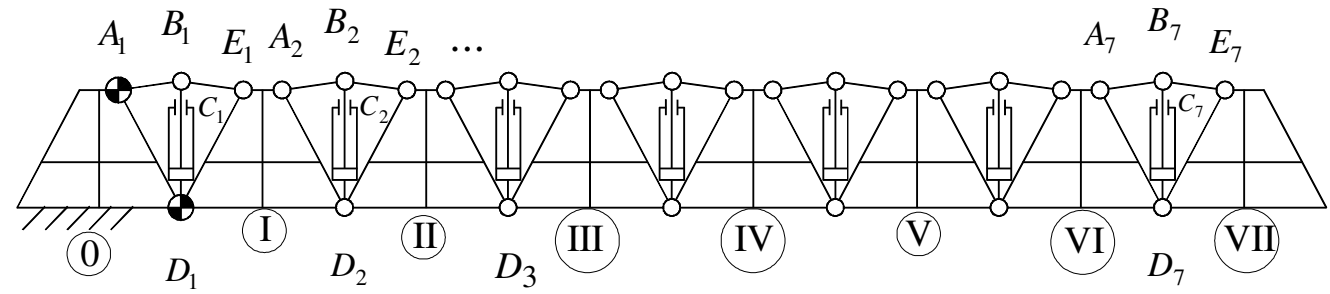

Fig. 1. Kinematic scheme of folding bridge consisting of eight segments

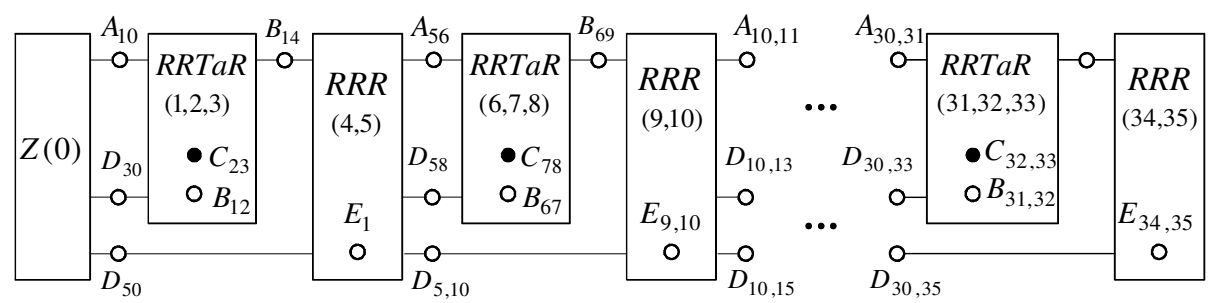

a)

$$
\begin{aligned}
Z(0)+R R T a R(1,2,3)+R R R(4,5 & +\operatorname{RRTaR}(6,7,8)+R R R(9,10)+\cdots \\
& +\operatorname{RRTaR}(31,32,33)+\operatorname{RRR}(34,35)
\end{aligned}
$$

b)

Fig. 2. Multipolar scheme and structural relation of folding bridge consisting of eight segments

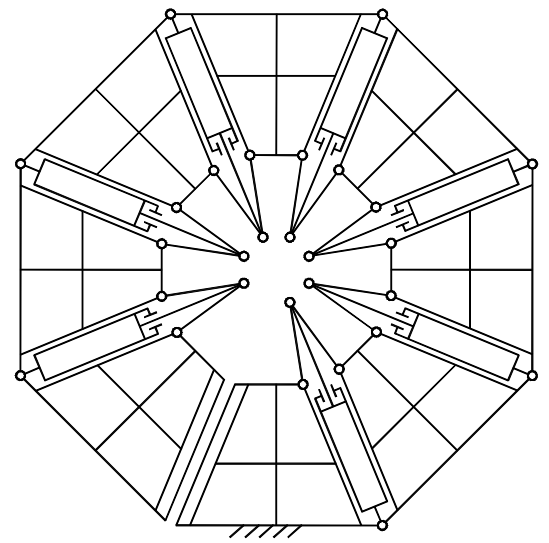

Fig. 3. Kinematic scheme of folding bridge in folded position

\section{Kinematic analysis of the folding bridge consisting of eight segments}

In the paper the kinematic analysis is made of the folding bridge consisting of eight segments, of which seven are mobile. Given that RRTaR motor dyads and $R R R$ dyads of aspect 1 are involved in the construction of the bridge, the corresponding modules will be studied for the analysis of the mechanism.

\subsection{Kinematic analysis of RRTaR motor dyad [3]}

Figure 4 shows the kinematic scheme of the $R R T_{a} R$ motor dyad. The input couplings are $A$ and $D$, and the inner couplings are $B$ and $C$. 


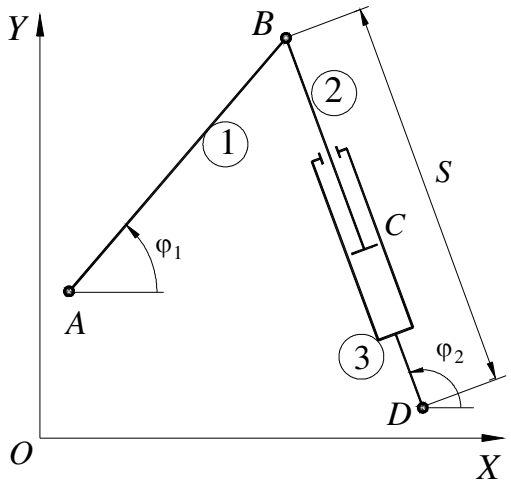

Fig. 4. Kinematic scheme of $R R T_{a} R$ motor dyad

In order to determine the kinematic parameters of the RRTaR motor dyad, knowing the following:

- $A B$ - length of element 1 ;

- $X A, Y A, X D, Y D$ - coordinates of input poles $A$ and $D$;

- $\quad \dot{X} A, \dot{Y} A, \dot{X} D, \dot{Y} D$ - projections of $A$ and $D$ poles linear velocities;

- $\quad \ddot{X} A, \ddot{Y} A, \ddot{X} D, \ddot{Y} D$ - projections of $A$ and $D$ poles linear accelerations;

- $S$ - independent parameter of active coupling $C$;

- $\quad \dot{S}$ - relative velocity of active coupling $C$;

- $\quad \ddot{S}$ - relative acceleration of active coupling $C$;

- $X B, Y B$ - approximate coordinates of the inner pole $B$,

we determine:

- $\varphi_{1}, \varphi_{2}$ - angles between $O x$ axis unit vector and $\overline{A B}$ and $\overline{D B}$ vectors;

- $\dot{\varphi}_{1}, \dot{\varphi}_{2}$ - angular velocities of elements 1 and 2 (3);

- $\ddot{\varphi}_{1}, \ddot{\varphi}_{2}$ - angular accelerations of elements 1 and 2 (3);

- $X B, Y B$ - exact coordinates of the inner pole $B$;

- $\dot{X} B, \dot{Y} B$ - projections of the linear velocity of pole $B$;

- $\quad \ddot{X} B, \ddot{Y} B$ - projections of the linear acceleration of pole $B$.

\subsection{Analysis of $R R T_{a} R$ dyad positions}

The system of position equations is obtained by projecting the vector equation

$$
\overline{O A}+\overline{A B}=\overline{O D}+\overline{D B}
$$

on the axes of the coordinate system, namely:

$$
\left\{\begin{array}{l}
A B \cdot \cos \varphi_{1}-S \cdot \cos \varphi_{2}-k=0 \\
A B \cdot \sin \varphi_{1}-S \cdot \sin \varphi_{2}-h=0
\end{array}\right.
$$

where $k=X D-X A, h=Y D-Y A$.

Solving the nonlinear system in the $\varphi_{1}$ and $\varphi_{2}$ unknowns is made by two methods, for each method a calculation procedure being developed.

The system of position equations (1), ninlinear, in the $\varphi_{1}$ and $\varphi_{2}$ unknowns, is solved by applying the Newton-Raphson iterative method, starting from a given initial solution.

The system solution for the iteration $(i+1)$ has the form:

$$
\left.\left\|\begin{array}{c}
\varphi_{1} \\
\varphi_{2}
\end{array}\right\|^{(i+1)}=\left\|\begin{array}{c}
\varphi_{1} \\
\varphi_{2}
\end{array}\right\|^{(i)}-W\left(\varphi_{1}^{(i)}, \varphi_{2}^{(i)}\right) \| \begin{array}{l}
f_{1}\left(\varphi_{1}^{(i)}, \varphi_{2}^{(i)}\right) \\
f_{2}\left(\varphi_{1}^{(i)}, \varphi_{2}^{(i)}\right)
\end{array}\right),
$$


where

$$
\begin{aligned}
f_{1}\left(\varphi_{1}, \varphi_{2}\right) & =A B \cos \varphi_{1}-S \cos \varphi_{2}+X A-X D \\
f_{2}\left(\varphi_{1}, \varphi_{2}\right) & =A B \sin \varphi_{1}-S \sin \varphi_{2}+Y A-Y D \\
W & =\left\|\begin{array}{cc}
-A B \sin \varphi_{1} & S \sin \varphi_{2} \\
A B \cos \varphi_{1} & -S \cos \varphi_{2}
\end{array}\right\|
\end{aligned}
$$

The initial solution:

$$
\begin{aligned}
& \varphi_{1}^{(0)}=\operatorname{ATAN} 2(Y B-Y A, X B-X A) ; \\
& \varphi_{2}^{(0)}=\operatorname{ATAN} 2(Y B-Y D, X B-X D),
\end{aligned}
$$

where $X B$ and $Y B$ - the approximate coordinates of point $B$ (they are input data).

The iterative process stops, when the difference of two consecutively calculated solutions is less than a $\varepsilon$ imposed (in the procedure we considered $\varepsilon=0.0001$ radians).

After determining the $\varphi_{1}$ and $\varphi_{2}$ unknowns, with the specified precision, the exact coordinates of point $B$ are calculated using the relations:

$$
\begin{aligned}
& X B=X A+A B \cos \varphi_{1} ; \\
& Y B=Y A+A B \sin \varphi_{1} .
\end{aligned}
$$

\subsection{Analysis of $R_{R T} R$ dyad velocities}

Derive the system of position equations in relation to time and obtain:

$$
\left\{\begin{array}{c}
-A B \sin \varphi_{1} \cdot \dot{\varphi}_{1}+S \sin \varphi_{2} \cdot \dot{\varphi}_{2}=A 1 \\
A B \cos \varphi_{1} \cdot \dot{\varphi}-S \cos \varphi_{2} \cdot \dot{\varphi}_{2}=A 2
\end{array}\right.
$$

where $\quad A 1=\dot{k}+\dot{S} \cos \varphi_{2}$;

$$
\begin{aligned}
& A 2=\dot{h}+\dot{S} \sin \varphi_{2} ; \\
& \dot{k}=\dot{X} D-\dot{X} A, \dot{h}=\dot{Y} D-\dot{Y} A .
\end{aligned}
$$

The system is linear in the $\dot{\varphi}_{1}$ and $\dot{\varphi}_{2}$ unknowns.

Apply the inverse matrix method and obtain:

$$
\left\|\begin{array}{l}
\dot{\varphi}_{1} \\
\dot{\varphi}_{2}
\end{array}\right\|=W^{-1}\|A 1\| .
$$

The projections of point $B$ linear velocity are:

$$
\begin{aligned}
& \dot{X} B=\dot{X} A-A B \sin \varphi_{1} \cdot \dot{\varphi}_{1} ; \\
& \dot{Y} B=\dot{Y} A+A B \cos \varphi_{1} \cdot \dot{\varphi}_{1} .
\end{aligned}
$$

\subsection{Analysis of RRTaR dyad accelerations}

Derive the system of velocity equations (3) in relation to time and obtain:

$$
\left\{\begin{array}{c}
-A B \sin \varphi_{1} \cdot \ddot{\varphi}_{1}+S \sin \varphi_{2} \cdot \ddot{\varphi}_{2}=B 1 \\
A B \cos \varphi_{1} \cdot \ddot{\varphi}_{1}-S \cos \varphi_{2} \cdot \ddot{\varphi}_{2}=B 2
\end{array},\right.
$$


where

$$
\begin{gathered}
B 1=\ddot{k}+\ddot{S} \cos \varphi_{2}-2 \dot{S} \dot{\varphi}_{2} \sin \varphi_{2}+A B \cos \varphi_{1} \cdot \dot{\varphi}_{1}^{2}-S \cos \varphi_{2} \cdot \dot{\varphi}_{2}^{2} ; \\
B 2=\ddot{h}+\ddot{S} \sin \varphi_{2}+2 \dot{S}_{\dot{\varphi}_{2}} \cos \varphi_{2}+A B \sin \varphi_{1} \cdot \dot{\varphi}_{1}^{2}-S \sin \varphi_{2} \cdot \dot{\varphi}_{2}^{2} ; \\
\ddot{k}=\ddot{X} D-\ddot{X} A ; \ddot{h}=\ddot{Y} D-\ddot{Y} A .
\end{gathered}
$$

The system is linear in the $\ddot{\varphi}_{1}$ and $\ddot{\varphi}_{2}$ unknowns, therefore:

$$
\left\|\begin{array}{l}
. . \\
\varphi_{1} \\
\ddot{\varphi_{2}}
\end{array}\right\|=W^{-1}\|B 1\| .
$$

The projections of point $B$ linear acceleration are:

$$
\begin{aligned}
& \ddot{X} B=\ddot{X} A-A B \cos \varphi_{1} \cdot \dot{\varphi}_{1}^{2}-A B \sin \varphi_{1} \cdot \ddot{\varphi}_{1} ; \\
& \ddot{Y} B=\ddot{Y} A-A B \sin \varphi_{1} \cdot \dot{\varphi}_{1}^{2}+A B \cos \varphi_{1} \cdot \ddot{\varphi}_{1} .
\end{aligned}
$$

The analysis of the dyads of aspect 1 , which intervene in the construction of the folding bridge mechanism, is similarly made.

\section{Results and discussion}

Each segment of the bridge is considered to have the shape of an isosceles trapezoid. The dimensions of the mechanism elements are: $\mathrm{A} 1 \mathrm{~B} 1=0.47 \mathrm{~m} ; \mathrm{B} 1 \mathrm{E} 1=0.47 \mathrm{~m} ; \mathrm{E} 1 \mathrm{~A} 2=0.3 \mathrm{~m}$; piston stroke $=0.540 \mathrm{~m} ; \mathrm{XA} 1=(\mathrm{E} 1 \mathrm{~A} 2) / 2 \mathrm{~m} ; \mathrm{YA} 1=0.9 \mathrm{~m} ; \mathrm{XD} 1=(\mathrm{D} 1 \mathrm{D} 2) / 2 \mathrm{~m} ; \mathrm{YD} 1=0 \mathrm{~m}$; linear velocity of the piston $=0.027 \mathrm{~m} / \mathrm{s}$; linear acceleration of the piston $=0 \mathrm{~m}$.

From the kinematic analysis of the mechanism the positions, velocities and accelerations of all the elements of the mechanism result.

Figure 5 shows the variation diagrams of angles $\varphi_{1}, \varphi_{3}, \varphi_{6}, \varphi_{8}, \varphi_{11}$ and $\varphi_{13}$, corresponding to the motor dyads in the first three segments. Similarly, the variation diagrams of the angles of the other elements of the folding bridge can be drawn.

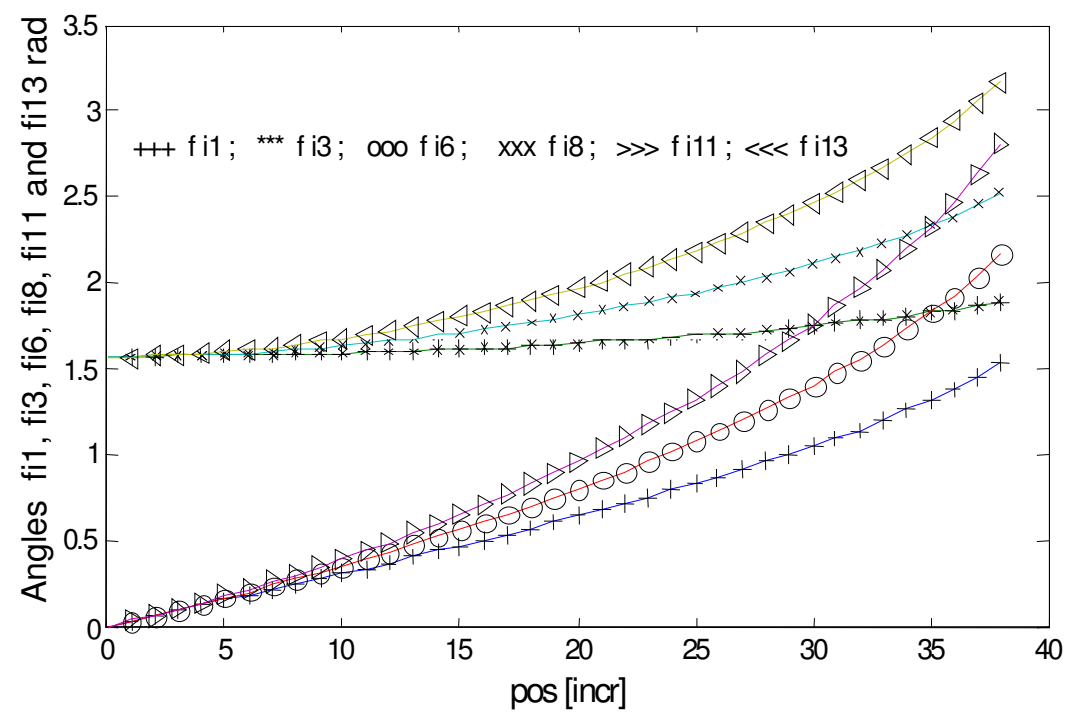

Fig. 5. Variation diagrams of angles $\varphi_{1}, \varphi_{3}, \varphi_{6}, \varphi_{8}, \varphi_{11}$ and $\varphi_{13}$

$\left(\varphi_{1}=\prec(\overline{A 1 B 1}, \overline{O X}), \varphi_{3}=\prec(\overline{D 1 B 1}, \overline{O X}), \varphi_{6}=\prec(\overline{A 2 B 2}, \overline{O X}), \varphi_{8}=\prec(\overline{D 2 B 2}, \overline{O X}), \ldots\right)$ 
Folding bridges are simple, easy-to-lay and easy to maintain. The construction of these bridges can be made from one or more segments, depending on the width of the water course. Both the kinematic study of the bridge mechanism and the kinetostatic study are based on the component modules, modules for which computational procedures have been prepared in different programming languages.

\section{Conclusions}

The kinematic parameters are presented in graphical form.

The linear transmission function was used to control the hydraulic cylinders. This transmission function can be combined with other transmission functions at the beginning and the end of the stroke. These transmission functions may be polynomial, with the polynomial degree equal to or higher than two, or trigonometric functions. Using combined transmission functions, the operation of the entire mechanism is quieter, with no start and stop shocks.

The kinematic analysis of the mechanism precedes its kinetostatic analysis, the kinematic joints reactions determination and motor forces inside the hydraulic cylinders.

As a result of the construction modularization, building such bridges is simple, with minimal cost and easy maintenance.

\section{Acknowledgement}

This work was funded by the Romanian Ministry of Research and Innovation, "SECTORIAL Program", (Contract no.3PS/02.11.2017 "Research to support the development of the capacity to assess and mitigate the impact of climate change and other stress factors on the state of forest ecosystems and wine-growing").

\section{References}

[1] Artobolevski I.I. Théorie des Mécanismes et des Machines (Theory of Mechanisms and Machines). Moscow: Ed. Mir, 1977. 453 p. (In French).

[2] Demidovich B., Maron A.I. Éléments de calcul numérique. $2^{\text {nd }}$ edition. Moscow: Ed. Mir, 1987. 717 p. (In French).

[3] Dorn W.S., Mc Cracken D.D. Metode numerice cu programe în FORTRAN IV. Bucureşti: Editura Tehnică, 1976. 468 p. (In Romanian).

[4] Duca C., Buium Fl., ş.a. Mecanisme. Iaşi: Editura Gh. Asachi, 2003. 481 p. (In Romanian).

[5] Moise V., Maican E., Moise Şt. I. Metode numerice în inginerie. Bucureşti: Ed. Bren, 2003. 305 p. (In Romanian).

[6] Moise V., Simionescu I., Ene M. etc. Analiza mecanismelor aplicate. Bucureşti: Editura Printech, 2008. 282 p. (In Romanian).

[7] Pelecudi Chr. Precizia mecanismelor. Bucureşti: Editura Academiei Republicii Socialiste Romania, 1975. 398 p. (In Romanian).

[8] Popescu I. Proiectarea mecanismelor plane. Institutul Politehnic Bucureşti, 1977, 577 p, (In Romanian).

[9] Pelecudi Chr., Maroş D., Merticaru V. etc. Mecanisme, Bucureşti: Editura Didactică şi Pedagogică, 1985. 394 p. (In Romanian).

[10] Simionescu I., Moise V. Mecanisme. Bucureşti: Editura Tehnică. 1999, 238 p. (In Romanian).

[11] Uicker J.J., Pennock G.R., Shigley J. E. Theory of Machines and Mechanisms. 4th Edition. New York: Oxford University Press, 2011. 928 p.

\section{Annex - related videos}

1. https://www.youtube.com/watch?v=x0Dj7XA77hw

2. https://www.youtube.com/watch?v=ftIpbQ4LiMY\&t=85s

3. https://www.youtube.com/watch? $\mathrm{v}=\mathrm{AtJCUihUvZc}$

4. https://www.youtube.com/watch?v=E5BF3Lvmi_8

5. https://www.youtube.com/watch?v=rOx_ZGLrgv4 